\title{
Autophagy: A New Phase in the Maturation of Growth Plate Chondrocytes Is Regulated by HIF, mTOR and AMP Kinase
}

\author{
Vickram Srinivas Jolene Bohensky Irving M. Shapiro \\ Department of Orthopaedic Surgery, Thomas Jefferson University, Philadelphia, Pa., USA
}

\section{Key Words}

Growth plate $\cdot$ Chondrocytes $\cdot$ Autophagy $\cdot$ AMP kinase $\cdot$ mTOR

\begin{abstract}
The overall goal of the investigation was to examine autophagy in the growth plate and to ascertain how this process was regulated. Herein, we show that in the postmitotic maturing zone of the growth plate, chondrocytes express an autophagic phenotype. This robust and particulate immunohistochemical response provides direct evidence that autophagy is a new and transient stage in the chondrocyte maturation pathway. We found that induction of autophagy was regulated by MTOR, a sensor of cellular metabolism. When mTOR was inhibited, changes in LC3 fluorescence indicated that this kinase regulated development of the autophagic state. To determine if AMP kinase was required for chondrocyte autophagy, we suppressed its expression in N1511 cells using siRNA technology. When these cells were serum starved, a condition that triggers autophagy, there was no change in LC3 distribution. This result confirmed that AMP kinase was required for the induction of the autophagic response. Based on the 2 studies described above, and our previous observation that HIF-1 is required for the induction of autophagy, we put forward the hypothesis that autophagy is regulated by the activities of AMP kinase and mTOR in
\end{abstract}

\section{KARGER}

Fax +41613061234

E-Mail karger@karger.ch

www.karger.com
(C) 2008 S. Karger AG, Basel

Accessible online at: www.karger.com/cto a HIF-1-dependent manner. Once autophagy is activated, the postmitotic chondrocytes would be expected to remain viable in their unique microenvironment and complete their life cycle.

Copyright $\odot 2008$ S. Karger AG, Basel

\section{Introduction}

The epiphyseal growth plate is a complex cartilaginous structure that directs long bone growth. Bone elongation is achieved by chondrocytes synthesizing an abundant extracellular matrix and undergoing hypertrophy; each of these activities promotes cartilage expansion, an interim state, in which the calcified cartilage matrix is eventually replaced by bone. One critical stage in the maturation process involves the regulated development of hypertrophy. During hypertrophy, the cells increase their size 3- to 5-fold, synthesize collagen type $\mathrm{X}$ and express matrix vesicles, small particles that serve as centers

Abbreviation used in this paper

PBS phosphate-buffered saline 
of mineralization [Anderson et al., 2005]. Interference with this process leads to abnormal cartilage formation, often loss of the fully differentiated phenotype and abnormalities in bone formation [Horton et al., 2007]. Terminally differentiated chondrocytes are deleted from the plate by the induction of apoptosis [Shapiro et al., 2005].

While the residence time of chondrocytes in the growth plate is limited in duration, the cells exist in a metabolically challenging microenvironment. A number of researchers have shown that the oxygen tension in the cartilage is low, and hence chondrocytes generate much of their metabolic energy through anaerobic glycolysis [Hatori et al., 1995; Shapiro et al., 2005]. We have argued that the environment may also activate an autophagic response in the maturing cells, which serves to maintain chondrocyte viability until the induction of apoptosis [Shapiro and Srinivas, 2007]. It is now evident that the autophagic response observed in many cell types serves a physiologic role in generating energy utilizing the cells' own protein and lipid stores [Klionsky, 2005]. It is also apparent that in many organisms and tissues, autophagy is a physiologic response and a requirement for normal development [Lum et al., 2005].

Surprisingly, little is known of the autophagic response in cartilage formation and long bone growth. In the present communication, we address this need by evaluating the growth plate for evidence of the induction of autophagy. In addition, we examine the mechanism of control of autophagy. We focus on the activities of 2 regulatory proteins, AMP kinase and mTOR. mTOR is a serine/ threonine kinase that has been implicated as a sensor of the nutrient status [Brugarolas et al., 2004], while AMP kinase senses the energy status of the cell [Hoyer-Hansen and Jaattela, 2007]. We show that maturing chondrocytes exhibit an autophagic phase and that regulation of the phase is dependent on the activities of $\mathrm{mTOR}$ and AMP kinase.

\section{Materials and Methods}

\section{Cell Culture}

N1511 mouse chondrocytes and its derived cell lines were cultured in $\alpha$-MEM (Invitrogen, Carlsbad, Calif., USA) containing $10 \%$ fetal bovine serum, $0.2 \% \mathrm{~L}$-glutamine, $0.2 \%$ penicillin/streptomycin and $0.2 \%$ sodium pyruvate. Cells were matured by the addition of BMP- 2 at a concentration of $100 \mathrm{ng} / \mathrm{ml}$. All cells were maintained at $37^{\circ} \mathrm{C}, 5 \% \mathrm{CO}_{2}$ and $95 \%$ air. Details of the method utilized and characterization of the mature phenotype are described elsewhere [Terkhorn et al., 2006]. In some experiments, mTOR function was inhibited using $25 \mathrm{nM}$ rapamycin (Calbiochem, Gibbstown, N.J., USA) for $2 \mathrm{~h}$.

Growth Plate Chondrocyte Autophagy
Detection of LC3 in Cartilage

Expression of LC3 was assessed immunohistochemically in longitudinal sections of the embryonic (E18.5) proximal tibial growth plate. When this microtubule-associated protein binds to vacuole membranes, its particulate organization provides a powerful biochemical marker for the induction of autophagy [Mizushima and Yoshimori, 2007]. Mice were sacrificed in accordance with ethical procedures approved by the Thomas Jefferson University IACUC. The tissue was decalcified and subsequently fixed in $4 \%$ paraformaldehyde in PBS ( $\mathrm{pH}$ 8.0). The fixed tissue was then paraffin embedded, serially sectioned $(5 \mu \mathrm{m})$ and permeabilized with proteinase $\mathrm{K}(10 \mu \mathrm{g} / \mathrm{ml})$. Next, serial sections were treated with an LC3 antibody (Abgent, San Diego, Calif., USA), at a dilution of $5 \mu \mathrm{g} / \mathrm{ml}$. Rabbit IgG $(5 \mu \mathrm{g} / \mathrm{ml})$ was used as a negative control. Following treatment with the primary antibody, sections were treated with Alexa Fluor 594-labeled secondary antibody.

Immunohistochemical Localization of LC3 in Chondrocytes in Culture

LC3 expression was assessed by immunohistochemistry [Bohensky et al., 2007a, b]. Cells were prepared at a density of 40,000 cells $/ \mathrm{ml}$ in a 24 -well plate and maintained in hypoxia or normoxia for $16 \mathrm{~h}$. After washing 3 times in phosphate-buffered saline (PBS), cells were fixed with $3.7 \%$ paraformaldehyde in PBS ( $\mathrm{pH}$ 8.0) for 10 $\mathrm{min}$ and then washed. The cells were then permeabilized with $0.5 \%$ Triton X-100 in PBS for $5 \mathrm{~min}$ and washed 3 times in PBS. Antigenic sites were blocked in $10 \%$ calf serum in PBS for $1 \mathrm{~h}$. After blocking, the cells were incubated with LC3 antibody at a dilution of 1:200 overnight at $4^{\circ} \mathrm{C}$. Subsequently, the treated cells were incubated with a fluorescein-labeled secondary antibody for $1 \mathrm{~h}$ at room temperature. The cells were then washed in PBS 3 times for $5 \mathrm{~min}$ and mounted in CrystalMount. Proteins were visualized by confocal microscopy (Fluoview; Olympus, Tokyo, Japan).

\section{siRNA Plasmid Construction}

An siRNA construction kit (Ambion) was utilized to downregulate the expression of AMP kinase. The following phosphorylated oligonucleotides were used to create the siRNAs: AMPK- $\alpha$ 1 (F) gatccgatcggccactacatcctgttcaagagacaggatgtagtggccgatcttttttggaaa and AMPK- $\alpha 1$ (R) agcttttccaaaaaagatcggccactacatcctgtctcttgaacaggatgtagtggccgatcg. Permanent cell lines were generated using $80 \%$ confluent monolayers transfected with the desired siRNA vector followed by clonal selection using $800 \mu \mathrm{g} / \mathrm{ml}$ hygromycin B (Invitrogen). Cell lines with backbone vector with scrambled sequences served as controls.

\section{Results}

Autophagic Response of Maturing Chondrocytes in the Growth Plate

Longitudinal sections of a mouse growth plate were prepared and stained for LC3, an indicator of autophagic vacuole formation. In the maturing zone, LC3-positive macroautophagic particles are evident. These fluorescent particles are either of low intensity or absent from proliferative chondrocytes as well as those exhibiting terminal differentiation (fig. 1). 
Fig. 1. LC3 expression in the mouse growth plate. Longitudinal section through a mouse growth plate stained with an antibody against LC3 and imaged using confocal microscopy. a Proliferating zone. Note the very low level of LC3 fluorescence. $\mathbf{b}$ Cells in the postproliferative maturing region. Intracellular particulate fluorescence (arrows) is characteristic of autophagic vacuole formation. There was minimal fluorescence in terminally differentiated chondrocytes.

Fig. 2. Effect of suppression of AMP kinase expression in N1511 cells on autophagy. AMP kinase expression in N1511 chondrocytes was silenced. These cells were serum starved for $4 \mathrm{~h}$ prior to staining with LC3. The control cells (pSHH) are dense and evidence particulate fluorescence, characteristic of autophagosome formation (arrows). In contrast, the silenced cells (siAMPK $\alpha$ ) are spread and, while diffusely stained with the antibody, fail to exhibit punctate fluorescence.

Fig. 3. Effect of inhibition of mTOR on autophagic activity. Cells were maintained in medium containing $10 \%$ serum and then stained with LC3. The mTOR-inhibited cells are condensed and exhibited punctate staining (arrows) characteristic of autophagosome formation. Control (untreated) cells in serum-containing medium were spread and did not display the autophagy phenotype (no punctate fluorescence).
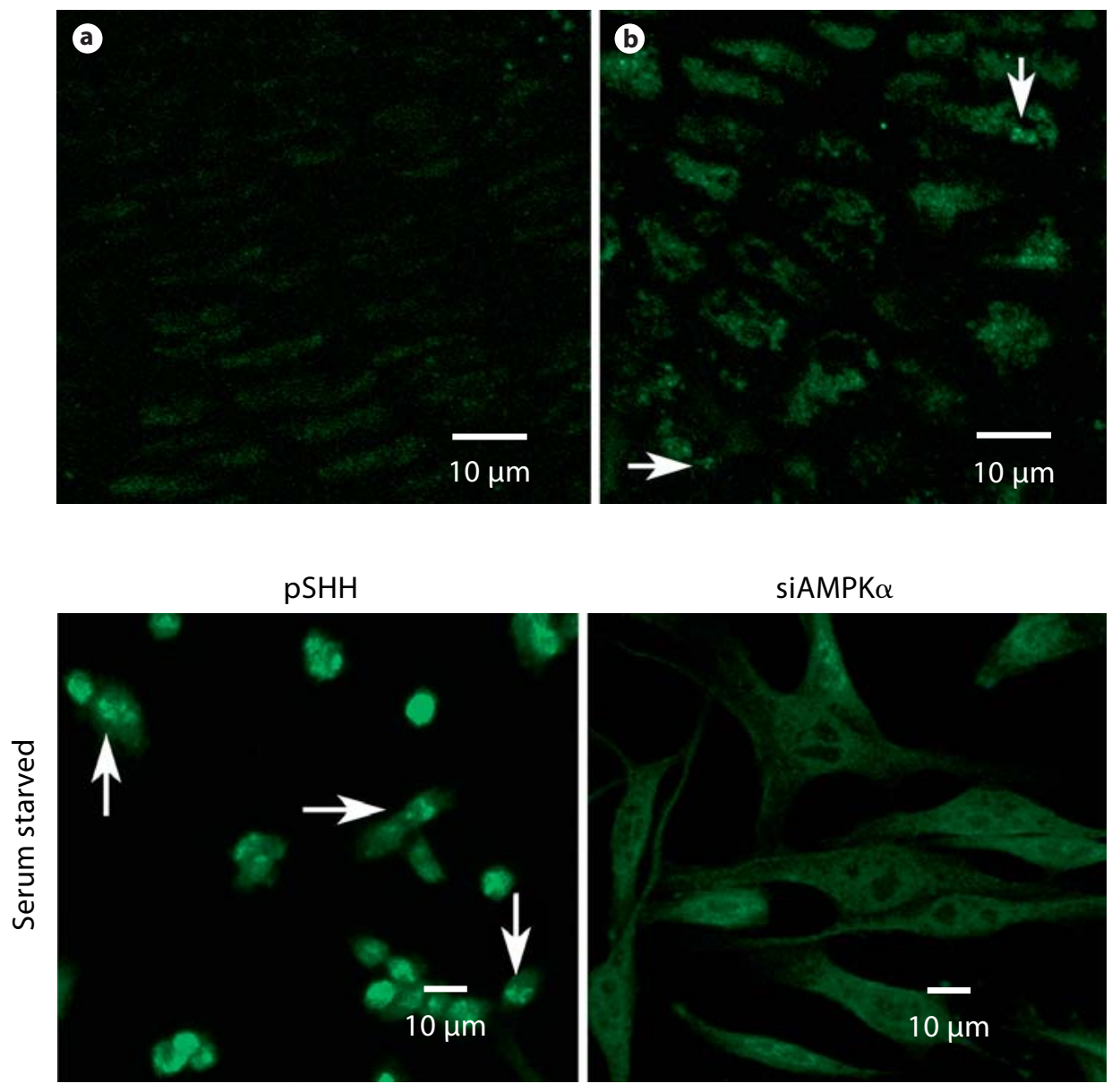

$\mathrm{pSHH}$

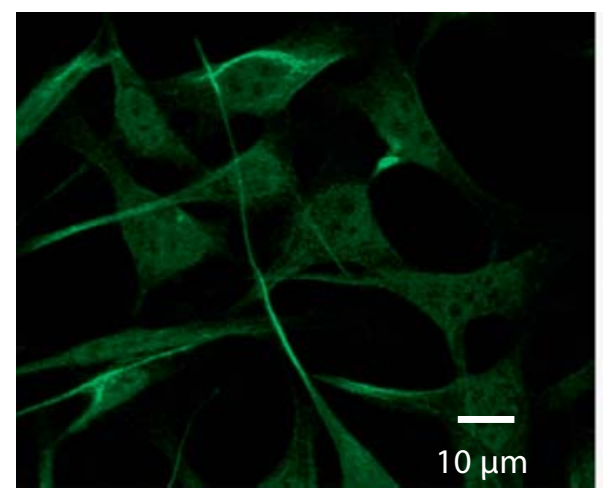

si mTOR

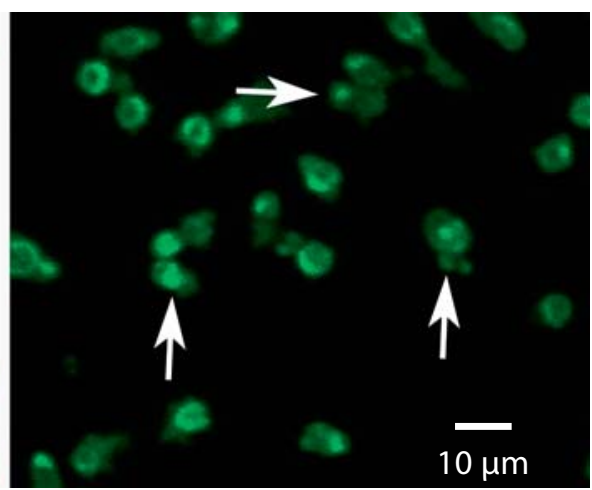

Functional AMP Kinase Is Required for Starvation-

Mediated Autophagy

Since autophagy is activated during nutritional and energy deprivation, we determined if the energy sensor, AMP kinase, was involved in the regulation of this process. AMP kinase-silenced cells were serum starved for
$4 \mathrm{~h}$ and then stained with LC3. Figure 2 shows that control cells (pSHH) evidence particulate fluorescence characteristic of autophagosome formation. In contrast, AMP kinase-silenced cells are spread and, while diffusely stained with the antibody, fail to exhibit the punctate fluorescence seen in autophagic cells. 
mTOR Inhibition Results in Constitutive Autophagy

Since AMP kinase suppresses mTOR activity, we determined the role of this protein in regulating the autophagic response. Cells were maintained in complete medium and treated with rapamycin, an agent that inhibits mTOR activity. The chondrocytes were then stained with an antibody against LC3. The mTOR-inhibited cells become condensed and exhibit punctate staining characteristic of autophagosome formation (fig. 3). In contrast, control cells in serum-containing medium are spread and LC3 staining is diffuse, indicating that these cells are not autophagic.

\section{Discussion}

The overall goal of the investigation was to examine autophagy in the growth plate and to ascertain how this process was regulated. Herein, we show that cells in the postmitotic maturing zone strongly express particulate LC3 fluorescence. This robust immunohistochemical response of the maturing chondrocyte provides direct evidence that autophagy is a new and transient stage in the chondrocyte maturation pathway. While we are cognizant that LC3 represents a single marker of the autophagic state, ongoing transmission electron-microscopic studies, as well as evaluation of beclin 1 expression and lysotracker fluorescence, lend further support to the notion that autophagosomes exist in growth plate chondrocytes, and as such, we infer that these organelles may play a functional role in the life history of the cell.

Since autophagy is a response to changes in the local environment, we evaluated the role of two microenvironmental sensors: AMP kinase and mTOR. For both of these agents, we determined their importance using genesilencing technology or a selective inhibitor. We found that induction of autophagy was regulated by mTOR, a sensor of the nutritional state of the cell [Brugarolas et al., 2004]. When mTOR was inhibited by rapamycin, there was extensive reorganization of LC3, indicating that the kinase regulated development of the autophagic state. Thus, mTOR served to block autophagy and inhibition of the kinase permitted chondrocytes to become autophagic. By downregulating the phosphorylation of targets S6K1 and 4E-BP1, mTOR integrates multiple signals, including those from nutrients, as well as metabolic signals from glycolysis and ATP [Plas and Thompson, 2005]. A number of studies have also shown a connection between mTOR and AMP kinase. Once activated, AMP kinase phosphorylates TSC2, which then suppresses mTOR.
From this perspective, mTOR serves as a nexus for growth factor, nutrient, $\mathrm{O}_{2}$ and energy signaling [Lum et al., 2005].

In addition to $\mathrm{mTOR}$, we investigated the relationship between AMP kinase and the induction of autophagy. AMP kinase senses the energy status of the cell and is exquisitely responsive to AMP [Savabi, 1994]. To determine if AMP kinase was required for chondrocyte autophagy, we suppressed AMP kinase expression in N1511 cells using siRNA technology. When these cells were serum starved, a condition that triggers autophagy, we failed to observe a loss of organization of LC3. In other words, if AMP kinase activity is blocked, autophagy cannot be activated in these chondrocytes. Based on these findings, we conclude that the two environmental sensors, AMP kinase and $\mathrm{mTOR}$, are required for the induction of the autophagic response.

Based on the studies described above, we put forward the hypothesis that the mechanism by which autophagy is controlled in chondrocytes is through the activities of two environmental sensors: AMP kinase and mTOR. Furthermore, we have previously shown that HIF-1 is required for the activation of autophagy [Bohensky et al., 2007b]. Also, since HIF-1 is a major metabolic regulator, we suggest that the autophagic activity regulated by AMP kinase and $\mathrm{mTOR}$ is mediated by this transcription factor. Increased HIF-1-mediated glycolytic activity would elevate AMP levels. The resulting AMP kinase activation and suppression of $\mathrm{mTOR}$ would cause increased autophagy. Once autophagy is activated, the postmitotic chondrocytes would be expected to remain viable in their unique microenvironment and complete their life cycle. Since autophagy can serve to delay the onset of apoptosis, it raised the following question: Is there a direct relationship between the induction of autophagy and apoptosis? Experiments are in progress to explore this interaction.

\section{Acknowledgements}

This work was supported by NIH grants DE 015694 and DE 016383 (V.S.) as well as DE 010875 and DE 013319 (I.M.S.). 


\section{References}

Anderson, H.C., R. Garimella, S.E. Tague (2005) The role of matrix vesicles in growth plate development and biomineralization. Front Biosci 10: 822-837.

Bohensky, J., I.M. Shapiro, S. Leshinsky, S.P. Terkhorn, C.S. Adams, V. Srinivas (2007a) HIF-1 regulation of chondrocyte apoptosis: induction of the autophagic pathway. Autophagy 3: 207-214.

Bohensky, J., I.M. Shapiro, S. Leshinsky, H. Watanabe, V. Srinivas (2007b) PIM-2 is an independent regulator of chondrocyte survival and autophagy in the epiphyseal growth plate. J Cell Physiol 213: 246-251.

Brugarolas, J., K. Lei, R.L. Hurley, B.D. Manning, J.H. Reiling, E. Hafen, L.A. Witters, L.W. Ellisen, W.G. Kaelin Jr (2004) Regulation of mTOR function in response to hypoxia by REDD1 and the TSC1/TSC2 tumor suppressor complex. Genes Dev 18: 2893 2904.
Hatori, M., C.C. Teixeira, K. Debolt, M. Pacifici, I.M. Shapiro (1995) Adenine nucleotide metabolism by chondrocytes in vitro: role of ATP in chondrocyte maturation and matrix mineralization. J Cell Physiol 165: 468-474.

Horton, W.A., J.G. Hall, J.T. Hecht (2007) Achondroplasia. Lancet 370: 162-172.

Hoyer-Hansen, M., M. Jaattela (2007) AMP-activated protein kinase: a universal regulator of autophagy? Autophagy 3: 381-383.

Klionsky, D.J. (2005) Autophagy. Curr Biol 15: R282-R283.

Lum, J.J., R.J. DeBerardinis, C.B. Thompson (2005) Autophagy in metazoans: cell survival in the land of plenty. Nat Rev Mol Cell Biol 6: 439-448.

Mizushima, N., T. Yoshimori (2007) How to interpret LC3 immunoblotting. Autophagy 3: 542-545.

Plas, D.R., C.B. Thompson (2005) Akt-dependent transformation: there is more to growth than just surviving. Oncogene 24: 74357442 .
Savabi, F. (1994) Interaction of creatine kinase and adenylate kinase systems in muscle cells. Mol Cell Biochem 133-134: 145-152.

Shapiro, I.M., C.S. Adams, T. Freeman, V. Srinivas (2005) Fate of the hypertrophic chondrocyte: microenvironmental perspectives on apoptosis and survival in the epiphyseal growth plate. Birth Defects Res C Embryo Today 75: 330-339.

-Shapiro, I.M., V. Srinivas (2007) Metabolic consideration of epiphyseal growth: survival responses in a taxing environment. Bone 40 : 561-567.

Terkhorn, S.P., J. Bohensky, I.M. Shapiro, E. Koyama, V. Srinivas (2006) Expression of HIF prolyl hydroxylase isozymes in growth plate chondrocytes: relationship between maturation and apoptotic sensitivity. J Cell Physiol 210: 257-265. 